\title{
Educación a distancia en situaciones de emergencia
}

\author{
Svenson, Nanette Archer \\ Centro de Investigación Educativa de Panamá (CIEDU) \\ Ciudad de Panamá, Panamá \\ nanette.svenson@gmail.com \\ Ruiz-Primo, María Araceli \\ Stanford University \\ Palo Alto, California, EEUU \\ aruiz@stanford.edu \\ Pacheco, Ivan \\ Boston College/Revista de Educación Superior en América Latina (ESAL) \\ Santiago, Chile \\ pacheciv@bc.edu \\ Reisberg, Liz \\ Boston College \\ Boston, Massachusetts, EEUU \\ reisberg@gmail.com
}

\section{Resumen}

Esta mesa redonda explora el proceso de transición de la docencia presencial a lo que se ha denominado educación a distancia (o educación remota) en situaciones de emergenciacomo la pandemia del COVID-19 y otras circunstancias de crisis-y las implicaciones correspondientes para las propuestas educativas durante el tiempo de la misma emergencia y para el futuro. Entre nuestros objetivos son la examinación de los siguientes sub-temas: 1) el contexto de Panamá y la clase de respuestas inmediatas implementadas en el proceso de transición a la educación a distancia durante COVID-19; 2) las implicaciones pedagógicas y organizativas para la educación remota con respecto a la evaluación; 3) los modelos de educación y docencia adoptados en las instituciones de educación superior en el contexto actual de emergencia sanitaria en América Latina y que significan para el futuro. Se exploran casos y ejemplos de diferentes países y distintas perspectivas. Factores importantes incluidas en la discusión, entre otros, son la existencia de una infraestructura digital; el modelo de organización escolar y la clase del liderazgo de los equipos docentes; el rol de la familia como agente educativo esencial para la educación primaria y secundaria; 
y la influencia del gobierno y los elementos políticos en la situación.

Palabras claves: educación a distancia/educación remota, emergencia, COVID-19, educación superior, América Latina.

\section{INTRODUCCIÓN}

Un estado de emergencia, provocado por una catástrofe natural, brote de enfermedad contagiosa, o conflicto político o civil, afecta e impide la vida normal de una comunidad, región o país. Casi siempre, los sistemas educativos sufren graves daños y trastornos. Brindar educación en estas situaciones a estudiantes de todos los niveles debe ser una prioridad nacional y una actividad de primera respuesta. Según a quién afecta la emergencia, se determina cómo responder para restaurar la entrega de instrucción, de una manera u otra. Generalmente, este esfuerzo involucra al gobierno, los organismos internacionales, el sector privado, y las comunidades afectadas. El tema de la educación en emergencias es complejo y ya reconocido como un campo de conocimiento especializado.

Este resumen extendido refleja la discusión presentada en la mesa redonda del mismo título durante el XVIII Congreso Nacional de Ciencia y Tecnología de la Asociación Panameña para el Avance de la Ciencia (APANAC XVIII). Explora el proceso de transición de la docencia presencial a la educación remota en situaciones de emergencia, como la pandemia del COVID-19 y otras crisis, y las implicaciones de esto para el futuro, en Panamá y en América Latina. La Dra. Nanette Archer Svenson del Centro de Investigación Educativa (CIEDU) presentó un breve panorama de la situación actual en Panamá. La Dra. María Araceli RuizPrimo de Stanford University en California habló sobre la adaptación evaluativa necesaria para la educación a distancia en emergencias. Y los Dres. Iván Pacheco y Liz Reisberg de Boston College en el estado de Massachusetts en los EEUU presentaron sobre la educación superior en la región latinoamericana durante la pandemia de COVID-19.

\section{LA EDUCACIÓN PANAMEÑA DURANTE LA PANDEMIA}

La República de Panamá tiene la desafortunada distinción de ser el país del mundo que acumula más días consecutivas sin educación presencial durante la pandemia actual, según informes recientes [1]. El país tiene 900,000 estudiantes, la gran mayoría (más de $80 \%$ ) de los cuales asisten a escuelas públicas, y desde que se detectó el primer caso de COVID-19 en marzo de 2020, el gobierno cerró las escuelas. Panamá no contaba con una plataforma oficial para la educación a distancia, pero en julio de 2020 el Ministerio de Educación (MEDUCA) anunció la reapertura de clases con programación remota apoyada por internet, televisión, radio y la distribución física de paquetes didácticos. En el último año, oficiales y docentes han trabajado para establecer las plataformas tecnológicas, guías y materiales digitales necesarios, capacitar a docentes y otros profesionales, e incorporar a familias 
al nuevo sistema, pero aún falta mucho por hacer según investigaciones recientes del UNICEF [2]. Los retos grandes incluyen la cobertura y costo de la conectividad, la capacidad tecnológica, el desempeño anterior, la calidad de materiales utilizados, el posicionamiento de los gremios docentes, y la voluntad política. Generalmente, se nota que la educación privada ha logrado más que la educación pública con respecto a la educación a distancia, y la educación superior ha logrado más que la educación primaria y secundaria, aunque existen excepciones a estas generalizaciones también.

Previo al estado actual de emergencia, Panamá enfrentaba un sistema educativo débil en términos de logros estudiantiles y otros indicadores de calidad. En las más recientes evaluaciones del Programa Internacional de Evaluación de los Alumnos (PISA) y del Estudio Regional Comparativo y Explicativo (ERCE), conducido por la UNESCO, los promedios de Panamá están muy por debajo de los del resto de los países. Igualmente, las recientes evaluaciones nacionales estandarizadas, CRECER, que miden el conocimiento de los estudiantes de tercer y sexto grados en Español, Matemáticas y Ciencias Naturales, muestran que la mitad del tercer grado no cuenta con conocimientos básicos en las dos primeras asignaturas y la mitad del sexto grado no ha logrado desarrollar sus competencias científicas. Además, en todas estas evaluaciones se nota que los promedios de los estudiantes de las escuelas privadas tienden a estar por encima de los de las escuelas públicas y se nota una tendencia similar con las poblaciones urbanas y rurales. La pandemia ha servido para resaltar las desigualdades inherentes en el sistema. Varios factores contribuyen al desequilibrio, pero entre los más importantes se encuentra el acceso a la conectividad y la tecnología. Durante este periodo de la pandemia, los estudiantes que han podido continuar con su educación son ellos que tienen una conexión al internet y un dispositivo electrónico. Lamentablemente, solamente el $40 \%$ de los estudiantes del sistema público tienen acceso al internet en sus hogares, y un $30 \%$ tienen acceso a una computadora. Para las familias que tratan de conectarse a través de teléfonos celulares, el costo de data es otro factor limitante. Al contrario, el $90 \%$ de los estudiantes de las escuelas privadas tienen acceso al internet y el $75 \%$ a una computadora [3].

Sin un estado de emergencia, las desigualdades entre las escuelas públicas y privadas panameñas, y entre las rurales y urbanas, tenían mucho que ver con los resultados educativos reportados; con la pandemia, es probable que estas desigualdades pueden ser la diferencia entre la continuación y el abandono de los estudios. La ausencia prolongada de la escuela y la desparedad entre la clase de educación remota entregada en los distintos centros educativos por todo el país amenazan la capacidad de aprendizaje actual y futura, y afectan a la salud mental de los estudiantes y las familias. El impacto de todo esto se dejará sentir en la próxima década, tanto en términos económicos como de salud pública. Actualmente, MEDUCA está en el proceso de orquestar un retorno gradual a las aulas, con protocolos de bioseguridad para las escuelas y previa vacunación de los educadores. Sin 
embargo, ha encontrado cierto grado de resistencia de los gremios docentes y el proceso ha llegado a estar altamente politizado. Cada semana más sin clases presenciales arriesga agravar la brecha educativa evidenciada en el país. Se espera que las lecciones y los logros tecnológicos del último año y medio sirvan para mejorar el sistema educativo, público y privado, en Panamá. Pero la realidad es que reparar la brecha educativa llevará años, y no bastará con volver a la situación pre-pandémica. La crisis sanitaria ha destapado el grado de la marginación de la mayoría de los estudiantes del sistema público y resolver este problema social va a requerir un esfuerzo aún más grande que el de enfrentar la pandemia.

\section{LA EDUCACIÓN REMOTA Y LA EVALUACIÓN}

La educación remota tiene implicaciones para los métodos tradicionales utilizados para la evaluación de estudiantes, maestros, instituciones, programas, políticas y sistemas. Durante un estado de emergencia, el contexto cambia por completo y por eso muchas de las referencias, indicadores y objetivos aplicados bajo condiciones normales no siguen siendo relevantes. Llega a ser crítico medir no solamente los resultados logrados sino también los procesos usados en el camino. La pandemia de COVID-19 de muchas maneras ha puesto en primer plano la importancia de comprender y documentar los procesos y contextos del aprendizaje y la necesidad de tomarlos en cuenta en el diseño e interpretación de evaluaciones [4].

Una pregunta crítica que debe hacerse acerca del aprendizaje de los estudiantes después del COVID-19 o en cualquier contexto de educación remota es qué han aprendido los estudiantes y cómo han aprendido. Las disparidades existente pre-pandemia se han exacerbado en los últimos meses y es importante entender cuáles son las condiciones de aprendizaje en las que los estudiantes han estado. Se presentaron argumentos que se enfocan en la necesidad de pruebas que sean más enfocadas en contenidos críticos, correctas técnicamente (interpretaciones válidas y justas, puntajes confiables), en sistemas balanceados que sean coherentes, comprensibles y continuos, en colectar información acerca de las oportunidades de aprendizaje que tienen los estudiantes para poder entender, triangular y contextualizar mejor los resultados de evaluación.

\section{COVID-19 Y LA EDUCACIÓN SUPERIOR EN AMÉRICA LATIN}

El coronavirus y su impacto en la educación superior de la región latinoamericana ha dominado las discusiones, buena parte de las noticias y la divulgación académica. El tránsito de la educación presencial a la educación remota es, sin duda, una característica que identificará esta época. Sin embargo, ha habido diferencias en la forma como el tema ha sido abordado en cada país. Unos países de la región han avanzado significativamente, 
gracias a la existencia de políticas claras con respecto a la oferta de educación virtual, mientras otros tuvieron que enfrentarse a la desconfianza generalizada en torno a la virtualidad en la educación superior.

Durante décadas se ha tratado de entender la diferencia entre educación presencial y educación a distancia. Con la irrupción de las Tecnologías de la Informática y las Comunicaciones (TIC), en particular la masificación de la internet, surgieron programas de educación virtual o en línea y con ellos un nuevo esfuerzo por diferenciar las modalidades y formas de entrega. Antes de la pandemia, la educación a distancia y en línea no gozaban de aceptación generalizada y en algunos casos se percibían como de baja calidad. En consecuencia, se limitaba el alcance de dichos estudios o la forma como los contenidos eran entregados. El caso de Perú ilustra esta situación. En su artículo 47, la Ley Universitaria dispone que "los estudios de pregrado de educación a distancia no pueden superar el 50\% de créditos del total de la carrera bajo esta modalidad" y que "los estudios de maestría y doctorado no podrán ser dictados exclusivamente bajo esta modalidad." Esta misma ley exige contar con título de doctorado obtenido de manera presencial para ocupar las siguientes posiciones: Miembro del Consejo Directivo de la Superintendencia Nacional de Educación Superior Universitaria, SUNEDU; Superintendente de la SUNEDU; Rector; Decano y Profesor Principal (Arts. 17.2.1, 20.1.2, 61.3; 69.3, 83.1). Incluso en Colombia, donde la educación superior a distancia y virtual han crecido de manera sostenida durante la última década, existía la percepción de que por ley no se podía ofrecer algunas carreras usando estas modalidades, al punto que cuando la pandemia se desencadenó, el Ministerio de Educación Superior tuvo que expedir una circular señalando de manera expresa que no existía prohibición para ofrecer programas de Derecho en línea [5].

Con la llegada de la pandemia, las cosas cambiaron. Se suspendieron las clases presenciales y en cuestión de semanas, las instituciones de Educación Superior (IES) y los gobiernos se volcaron a las TIC para impartir clases y ofrecer contenidos. Varios gobiernos a lo largo y ancho del planeta introdujeron modificaciones temporales a sus normatividades para autorizar la oferta de programas de educación presencial utilizando mediaciones electrónicas, tales como video conferencias, chats, correo electrónico y muchas otras. Era educación presencial, pero ofrecida a distancia. Ahora, el interés por diferenciarse correspondió a quienes ofrecían y sabían de educación virtual y a distancia. Era importante marcar una clara diferencia entre educación virtual y el tipo de enseñanza que a raíz de la pandemia se estaba ofreciendo. Así, cobró importancia el concepto de "enseñanza remota en emergencia", definida como: "un cambio temporal en la entrega de instrucción a un modo de entrega alternativo debido a circunstancias de crisis. Implica el uso de soluciones de enseñanza totalmente remotas para la instrucción o la educación que de otro modo se impartirían de forma presencial o como cursos semipresenciales o híbridos y que volverán a ese formato una vez que la crisis o la emergencia haya disminuido." [6]. 
Durante la crisis del COVID-19, las IES demostraron su capacidad para participar en la respuesta integral a una crisis de magnitud global. Su articulación con otros actores sociales fue diferente de un lugar a otro, pero, en general, participaron en los esfuerzos de mitigación y control de la pandemia y procuraron extender sus servicios más allá de sus estudiantes y empleados, buscando beneficiar a las comunidades de sus áreas de influencia. Resulta que el entorno de las IES se está transformando a una velocidad inimaginable. La pandemia sacó la universidad de su tendencia hacia un estado de complacencia con el status quo y la forzó a buscar nuevas formas de entrega, colaboraciones, y sistemas para asegurar la calidad de la instrucción. Ha despertado una disposición hacia el cambio y nuevas posibilidades. Entre las lecciones aprendidas durante COVID-19 se puede destacar las siguientes:

1. Cualquiera puede aprender a dictar una clase virtual, pero no una de calidad. La educación virtual de calidad exige mucha preparación, capacitación, un equipo multidisciplinario, y contenidos muy estructurados. Ante la creciente importancia de las nuevas formas y mediciones de aprendizaje, el diseño de materiales y evaluaciones adecuadas cobra mayor pertinencia.

2. La tecnología necesaria existe hace tiempo y avanza a pasos agigantados. No hay vuelta atrás. El mundo ya entiende que sí es posible un modelo de enseñanza y de trabajo en el que se hace uso extensivo de la tecnología, afectando profundamente la interacción entre las personas.

3. Las desigualdades presentes en condiciones ordinarias se magnifican en situaciones de emergencia. La falta de recursos financieros, espacio físico, y acceso a servicios públicos marcan importantes diferencias, y estas diferencias se ampliarán si no dirigimos atención a ellas.

4. Hay que preguntarse si tiene sentido seguir diferenciando la educación tradicional de la virtual. Es necesario remover barreras artificiales tales como la exclusión de acceso a cargos públicos o privados como consecuencia de haber cursado estudios en programas no presenciales. La tendencia es hacia modelos híbridos y personalizados, en proporciones y formas diferentes.

5. Hay que estar preparados. Los países, IES y estudiantes que tenían los conocimientos y recursos adecuados para utilizar la tecnología efectivamente afrontaron la crisis de manera mucho menos traumática. La mejor manera de preparase para usar las nuevas tecnologías es usándolas e incorporándolas en la vida cotidiana. También, las IES y los gobiernos deben incluir el concepto de riesgo en sus estrategias de planeación para poder manejar emergencias futuras.

6. Las colaboraciones (internas y externas) pueden fortalecer las IES y contribuir a sus capacidades de enfrentar situaciones de emergencia. La cooperación entre departamentos e instituciones sirve para fomentar el intercambio de ideas, el desarrollo de soluciones a problemas compartidos, y la maximización de 
conocimientos y habilidades complementarias. Ahora la pregunta es cómo mantener e institucionalizar el aprendizaje de la pandemia para que contribuya a un desarrollo continuo de las IES.

\section{CONCLUSIONES}

Un estado de emergencia tiende a poner en relieve las deficiencias preexistentes en el sistema educativo. La pandemia actual ha empujado por toda la región y a todos los niveles una conciencia sobre las inequidades existentes, la subutilización de nuevas tecnologías y la falta de capacitación docente al respecto, mallas curriculares anticuadas, y sistemas de evaluación inadecuadas. Ha concentrado más atención en la importancia del aprendizaje, el contenido crítico, las limitaciones de la clase magistral, y las circunstancias y condiciones de los estudiantes y los docentes. Ha provocado nuevas colaboraciones una disposición de compartir ideas, y mucha innovación. ¡Ojalá que aprovechemos de lo que hemos experimentado durante el último año para mejorar el futuro de la educación a todos los niveles!

\section{Referencias}

[1] R. De Hoyos, Rafael y J. Saavedra, Jaime, "Banco Mundial Blogs: Es hora de volver a aprender." https://blogs.worldbank.org/es/education/es-hora-de-volver-aprender.

[2] UNICEF, "Situación de las Familias con Niños, Niñas y Adolescentes durante la pandemia por COVID-19 en Panamá - Tercera Encuesta de Hogares, Junio 2021." https://www.unicef.org/panama/ informes/tercera-encuesta-de-hogares.

[3] Centro de Investigación Educativa (CIEDU), “https://ciedupanama.org/.

[4] National Academy of Education, "Educational Assessments in the COVID-19 Era and Beyond." https://naeducation.org/wp-content/uploads/2021/02/Educational-Assessments-in-the-COVID-19Era-and-Beyond.pdf.

[5] Observatorio de la Universidad Colombiana, https://www.universidad.edu.co/.

[6] T.S. Hodges, C. Kerch, C. and M. Fowler, "Teacher Education in the Time of COVID-19: Creating Digital Networks as University-School-Family Partnerships. Middle Grades Review, 6 (2), 2020. https://scholarworks.uvm.edu/mgreview/vol6/iss2/4.

\section{Autorización y Licencia CC}

Los autores autorizan a APANAC XVIII a publicar el artículo en las actas de la conferencia en Acceso Abierto (Open Access) en diversos formatos digitales (PDF, HTML, EPUB) e integrarlos en diversas plataformas online como repositorios y bases de datos bajo la licencia CC:

Attribution-NonCommercial-ShareAlike 4.0 International (CC BY-NC-SA 4.0) https://creativecommons.org/licenses/ by-nc-sa/4.0/.

Ni APANAC XVIII ni los editores son responsables ni del contenido ni de las implicaciones de lo expresado en el artículo. 